\title{
Instillation of bradykinin into femoral artery elicits cardiorespiratory reflexes involving perivascular afferents in anesthetized rats
}

\author{
SANJEEV K. SINGH* (D, M. B. MANDAL and R. RAVINDRAN
}

Department of Physiology, Institute of Medical Sciences, Banaras Hindu University, Varanasi, UP, India

Received: October 12, 2019 • Accepted: January 07, 2020

Published online: April 14, 2020

(c) 2020 Akadémiai Kiadó, Budapest

\begin{abstract}
The physiology of baroreceptors and chemoreceptors present in large blood vessels of the heart is well known in the regulation of cardiorespiratory functions. Since large blood vessels and peripheral blood vessels are of the same mesodermal origin, therefore, involvement of the latter in the regulation of cardiorespiratory system is expected. The role of perivascular nerves in mediating cardiorespiratory alterations produced after intraarterial injection of a nociceptive agent (bradykinin) was examined in urethane-anesthetized male rats. Respiratory frequency, blood pressure, and heart rate were recorded for $30 \mathrm{~min}$ after the retrograde injection of bradykinin/saline into the femoral artery. In addition, paw edema was determined and water content was expressed as percentage of wet weight. Injection of bradykinin produced immediate tachypneic, hypotensive and bradycardiac responses of shorter latency (5-8 s) favoring the neural mechanisms involved in it. Injection of equi-volume of saline did not produce any responses and served as time-matched control. Paw edema was observed in the ipsilateral hind limb. Pretreatment with diclofenac sodium significantly attenuated the bradykinin-induced responses and also blocked the paw edema. Ipsilateral femoral and sciatic nerve sectioning attenuated bradykinin-induced responses significantly, indicating the origin of responses from the local vascular bed. Administration of bradykinin in the segment of an artery produced reflex cardiorespiratory changes by stimulating the perivascular nociceptors involving prostaglandins. This is a novel study exhibiting the role of peripheral blood vessels in the regulation of the cardiorespiratory system.
\end{abstract}

\section{KEYWORDS}

nociceptive agent, bradykinin, diclofenac sodium, vasosensory afferents, perivascular nerve, femoral and sciatic denervation, VR1 receptors

\footnotetext{
* Corresponding author. Tel.: +91 7897091535; Fax: +91 542-2367568/2368174, E-mail: drssks@gmail.com
} 


\section{INTRODUCTION}

Sensory nerves innervating the peripheral blood vessels have been implicated in the pain associated with migraine, angina, embolism, intermittent claudication and myocardial infarction. Peripheral vascular disorders are implicated in long-term cardiovascular alterations and other behavioral changes $[9,10]$. Since peripheral blood vessels and large blood vessels of the heart are of similar mesodermal origin, therefore it was proposed that peripheral blood vessels are involved in sensing and signaling the perivascular environmental information to the CNS, regulating systemic cardiorespiratory responses. From the previous work conducted on the rat model, it could be inferred that chronic disorders like diabetes mellitus and hypertension are characterized by widespread degeneration of the perivascular afferent neurons. This would lead to reduced release of vasodilator substances in the vicinity contributing to increased peripheral vascular resistance, but the patho-physiological significance of this ideology has to be further investigated $[16,29]$.

It has been shown elsewhere that intra-arterial (i.a.) injection of capsaicin $/ \alpha \beta$ MeATP produces immediate hyperventilatory and hypotensive responses $[18,27]$ indicating the role of peripheral blood vessels in the modulation of cardiorespiratory responses. In a separate series of work, it has been shown in our laboratory that i.a. injection of Mesobuthus tamulus (BT) venom elicits reflex cardiorespiratory changes, which last longer than pure chemical agonist-induced cardiorespiratory changes [24-26].

Since the BT venom is a mixture of several nociceptive agents such as polypeptides, bradykinin, histamine, prostaglandins, 5 -HT, etc., $[1,4,5,13,19,21,30]$ hence, it became necessary to identify the effect of individual components of the venom on vasosensory reflex responses. Bradykinin is one of the potent nociceptive components of the venom, therefore it was chosen for the elicitation of the vasosensory reflex responses. In a study elsewhere, it has been shown that i.a. injection of bradykinin produces hyperventilatory and hypotensive responses, but changes in heart rate (HR) were not observed in this study, and the observation period was also short [28]. In the present study, changes in the HR were also observed along with the changes in respiratory frequency (RF) and mean arterial pressure (MAP) for an extended period of time (30 min), which enables us to understand the complete changes occurring in the cardiorespiratory system.

Therefore, this study was planned to evaluate the role of peripheral blood vessels in producing the reflex cardiorespiratory responses by using bradykinin, in anesthetized rats. The experimental model was designed in such a way that the nociceptive agonist was instilled into a local segment of the femoral artery, so that the vasosensory afferents could be excited precisely and the mechanism could be ascertained.

\section{MATERIALS AND METHODS}

All the chemicals (saline/agonist/antagonist) were instilled into the femoral artery retrogradely and six animals were utilized in each group. The volume of chemicals was kept constant $(0.10$ $\mathrm{mL}$ ) for all injectables and the temperature of the lab was maintained at $25 \pm 2{ }^{\circ} \mathrm{C}$.

\section{ANIMALS AND ANESTHESIA}

Ethical clearance was obtained from the Institute Animal Ethical Committee, Banaras Hindu University before the commencement of the animal experiments. All the animals were used in 
accordance with the US National Research Council's "Guide for the Care and Use of Laboratory animals." Animals were exposed to 12:12 h light/dark cycle and food/water was provided ad libitum (Hindustan Lever Ltd, Mumbai). Urethane (Merck, Germany) was dissolved in double distilled water in the concentration of $0.5 \mathrm{~g} / \mathrm{mL}$. Healthy male albino rats (CharlesFoster strain; $235.34 \pm 10.56 \mathrm{~g}$ ) were anesthetized with an intra-peritoneal injection of urethane $(1.5 \mathrm{~g} / \mathrm{kg})$. A maintenance dose $(50-100 \mathrm{mg})$ of anesthesia was given as per the requirement.

\section{DISSECTION AND RECORDING}

Under the effect of anesthesia, tracheostomy was performed to keep the respiratory tract patent. A polyethylene tube of appropriate size was inserted in the trachea and secured tightly. The length of the tracheostomy tube was kept less to minimize the dead space. Tracheal secretions were aspirated as per the requirement. At the end of the experiments, animals were sacrificed by overdose of anesthesia. The femoral artery was dissected in the femoral triangle and skeletonized. The right femoral artery was cannulated proximally by a pediatric i.v. cannula with injection valve $(24 \mathrm{G}$, double ported) filled with heparinized saline $(20 \mathrm{IU} / \mathrm{mL})$. This cannula was used for BP recording as well as for the administration of drugs (saline/agonist/antagonists), as it has a double port (Fig. 1). The single cannulation for BP monitoring and drug administration minimizes the surgical injury to the animal. It also prevents systemic spillage, as the chemicals are injected against the flow of blood, turning the cannulated femoral artery into an end artery. Another advantage of drug administration via the same route (BP recording route) is that it marks the stimulus artifact by interrupting the BP recording for a fraction of second. We also avoided the cannulation of the common carotid artery, as it may compromise the circulation of pontomedullary areas which regulate the cardiorespiratory reflexes. Further, the cannula was connected to a pressure transducer, which in turn was connected to a bridge amplifier and finally to the data acquisition system (Power Lab 26 T, AD Instruments, Australia), which provides the mean arterial pressure on the personal computer (PC; Fig. 1).

The respiratory movements were recorded by securing the skin over the xiphisternum and connecting it to a force transducer via a thread. The electrocardiographic (ECG) potentials were recorded by using needle electrodes, connected in standard limb lead-II configuration. The blood pressure (BP), respiratory movements and ECG were recorded on a PC connected to the data acquisition system (Fig. 1). The respiratory frequency (RF), mean arterial pressure (MAP) and heart rate (HR) were obtained from the original recordings of the experiments. The latent period for all these parameters at various concentrations was also calculated from original recordings.

\section{DETERMINATION OF PAW EDEMA}

At the end of experiments, both the hind paws were disarticulated at the level of the ankle joint and the skin was cut open and removed. It was then weighed and dried in an electric oven (90 ${ }^{\circ} \mathrm{C}$ ) for a period of $72 \mathrm{~h}$. The difference in wet weight and dry weight of a paw provided the water content and was expressed as the percentage of wet weight. 


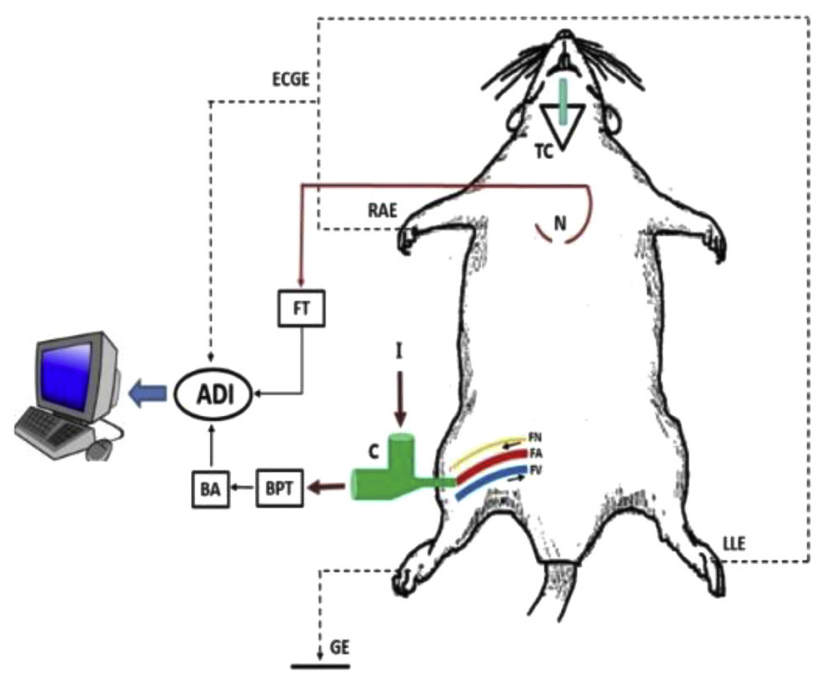

Figure 1. Schematics of the rat showing the experimental design for the recording of cardiorespiratory parameters. $\mathrm{ECGE}=$ Electrocardiogram electrodes; $\mathrm{TC}=$ Tracheal cannula; $\mathrm{N}=$ Needle to secure the skin for recording of respiratory movements; RAE $=$ Right arm electrode; LLE $=$ Left leg electrode; FT $=$ Force transducer; $\mathrm{ADI}=\mathrm{AD}$ Instrument for data acquisition (Power lab $26 \mathrm{~T}$, Australia); $\mathrm{BA}=$ Bridge amplifier; $\mathrm{BPT}=$ Blood pressure transducer, $\mathrm{C}=$ Cannula (double ported, $24 \mathrm{G}$ ); I = Injection port for drugs/saline with injection valve; $\mathrm{FN}=$ Femoral nerve; $\mathrm{FA}=$ Femoral artery; $\mathrm{FV}=$ Femoral vein; $\mathrm{GE}=$ Grounding electrode

\section{EXPERIMENTAL PROTOCOL}

\section{Determination of concentration-response of bradykinin}

After dissection, $30 \mathrm{~min}$ was given to the animals for the stabilization of cardiorespiratory parameters. Then, the initial recordings of respiration, BP and ECG were performed. Normal saline $(0.10 \mathrm{~mL})$ was injected into the femoral artery and the recordings of the cardiorespiratory parameters were made for $15 \mathrm{~min}$ at 5-min intervals. Next, bradykinin (10 nM, $100 \mathrm{nM}$ and 1 $\mu \mathrm{M}$ in $0.10 \mathrm{~mL}$, i.a.) was injected and cardiorespiratory responses were recorded for $30 \mathrm{~min}$ at 5min intervals. At the end of the experiment, both the hind paws were disarticulated from the ankle joint for estimation of paw edema.

\section{Effect of diclofenac sodium on bradykinin-induced cardiorespiratory changes}

After stabilization of the animal, the initial recordings of respiration, BP and ECG were performed. Diclofenac sodium (NSAID, $1 \mathrm{mg} / \mathrm{kg}$ ) was injected into the femoral artery and the cardiorespiratory parameters were recorded for $20 \mathrm{~min}$ to see if diclofenac sodium causes any alteration in the cardiorespiratory parameters. Equi-volume $(0.1 \mathrm{~mL})$ of normal saline was injected into the femoral artery and cardiorespiratory parameters were recorded for 15 min at 5min intervals. It was also observed that a gap of $20 \mathrm{~min}$ was sufficient to produce the antiinflammatory effects of the drug. Then, bradykinin $(1 \mu \mathrm{M}, 0.10 \mathrm{~mL}$, i.a.) was injected in these animals and the cardiorespiratory parameters were recorded for $30 \mathrm{~min}$ at 5 -min intervals. 


\section{Effect of neurotomy on bradykinin-induced cardiorespiratory changes}

The initial recordings of BP, ECG and respiration were performed. The sciatic and femoral nerves on the ipsilateral side were sectioned and cardiorespiratory parameters were recorded for $15 \mathrm{~min}$ at every $5 \mathrm{~min}$. Equi-volume $(0.10 \mathrm{~mL})$ of normal saline was injected into the femoral artery and cardiorespiratory parameters were recorded for $15 \mathrm{~min}$ at 5-min intervals. Bradykinin in the concentration of $1 \mu \mathrm{M}$ was injected in these animals into the femoral artery retrogradely, and the cardiorespiratory parameters were recorded for $30 \mathrm{~min}$ at 5-min intervals.

\section{Effect of normal saline on cardiorespiratory changes}

In a separate group of experiments, normal saline $(0.10 \mathrm{~mL}$.) was injected into the same femoral artery and the cardiorespiratory responses (RF, BP and HR) were recorded for $30 \mathrm{~min}$ at the interval of $5 \mathrm{~min}$. These animals served as a time-matched control group for the other sets of experiments. Paw edema was also determined in these animals.

\section{DRUGS AND SOLUTIONS}

Urethane was procured from Merck, Germany and was freshly dissolved in double distilled water $(0.5 \mathrm{~g} / \mathrm{mL})$ before each experiment. Bradykinin acetate salt was procured from the Sigma Chemicals Company, St Louis, USA. Stock solution of bradykinin $(1 \mathrm{mg} / \mathrm{mL})$ was prepared in double distilled water and kept in refrigerator. Subsequent dilutions were made in normal saline at the time of the experiments. The volume of all the drugs was kept to a minimum and constant $(0.10 \mathrm{~mL})$ to minimize stretch-induced responses. Diclofenac sodium was obtained from Cipla Pharmaceutical Company, Mumbai, India. Heparin $(1,000 \mathrm{U} / \mathrm{mL})$ was obtained from Biological Evans Ltd, Hyderabad, India.

\section{STATISTICAL ANALYSIS}

The results were presented as mean \pm SEM values. The statistical significance between two groups was analyzed by comparing the RF, MAP and HR responses of bradykinin groups with the saline group. The comparisons of various groups were made by Student's $t$-test for paired observations and Post-Hoc correction using Dunnett's $t$-test (two sided) for other observations by SPSS-16.0 software. A $P$ value $<0.05$ was considered significant.

\section{RESULTS}

Intra-arterial (i.a.) injection of bradykinin $(0.10 \mathrm{~mL})$ into the femoral artery produced immediate tachypneic, hypotensive and bradycardiac responses within 5-8 s. Injection of equi-volume of saline $(0.10 \mathrm{~mL})$ did not produce any changes in RF, MAP and HR for 30 min and served as time-matched control (Figs 2 and 3). Saline was also injected in each experiment 15 min prior to the injection of bradykinin, and no changes were observed in the cardiorespiratory parameters (Fig. 3). 


\section{Effect of bradykinin on respiratory frequency}

Bradykinin-induced respiratory changes were concentration-dependent: they were minimal with $10 \mathrm{nM}$ and maximal with $1 \mu \mathrm{M}$ concentration. After the injection of bradykinin, there was an immediate increase in rate of respiration (20-40 per min) at all concentrations of bradykinin with latency of 5-8 s (Figs 2 and 3). The increase in RF was significantly greater than in the time-matched saline group $[P<0.05$, Post-Hoc correction using Dunnett's $t$-test (two sided) $]$ or as compared to the corresponding initial value at all concentrations of bradykinin $(P<0.05$, Student's $t$-test for paired observations). The response was short-lived and lasted for a few seconds, then returned to the initial level within $5 \mathrm{~min}$, and subsequently remained at that level up to $30 \mathrm{~min}$. The tachypneic response was maximal with $1 \mu \mathrm{M}$ concentration of bradykinin with the shortest latency of $4.92 \pm 0.35 \mathrm{~s}$ (Figs 2 and 3).

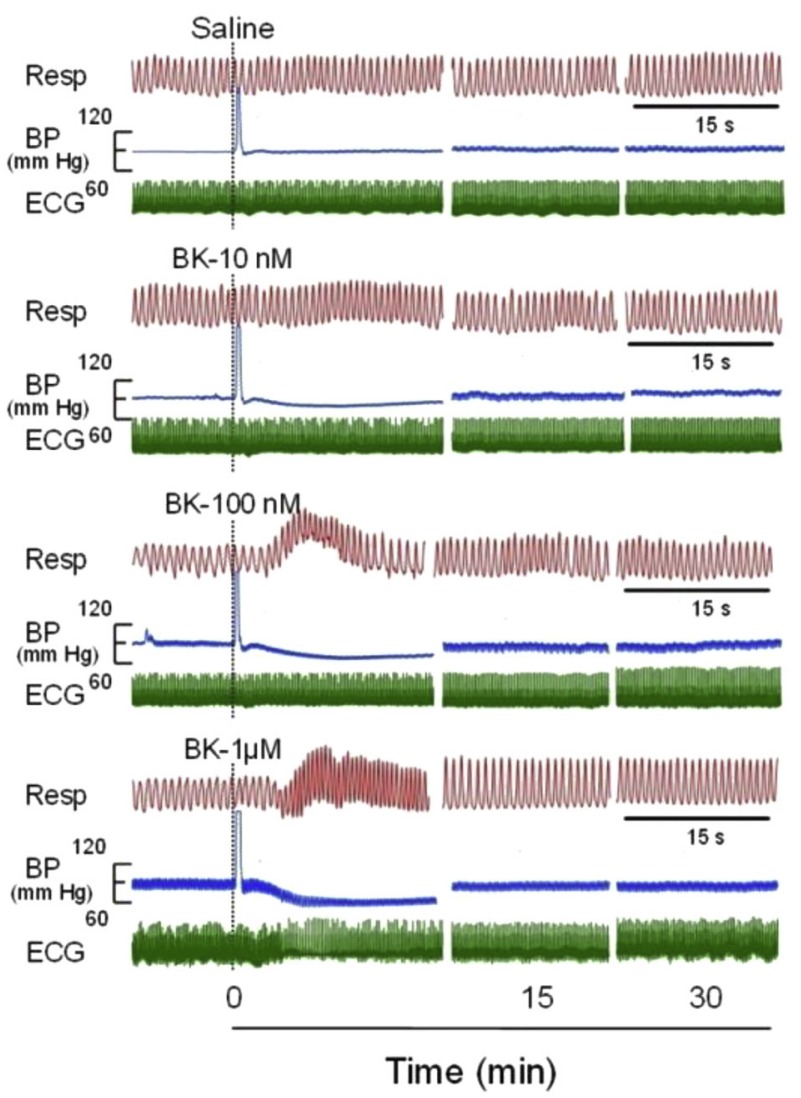

Figure 2. Original recordings showing the effect of different concentrations of bradykinin (BK) on respiration (Resp), blood pressure (BP) and electrocardiogram (ECG) as compared with the effect of normal saline. The responses after bradykinin/saline are shown at different time intervals as indicated in the lower panel. The point of injection is shown by dotted line. The horizontal line in each panel is $15 \mathrm{~s}$ for all parameters 

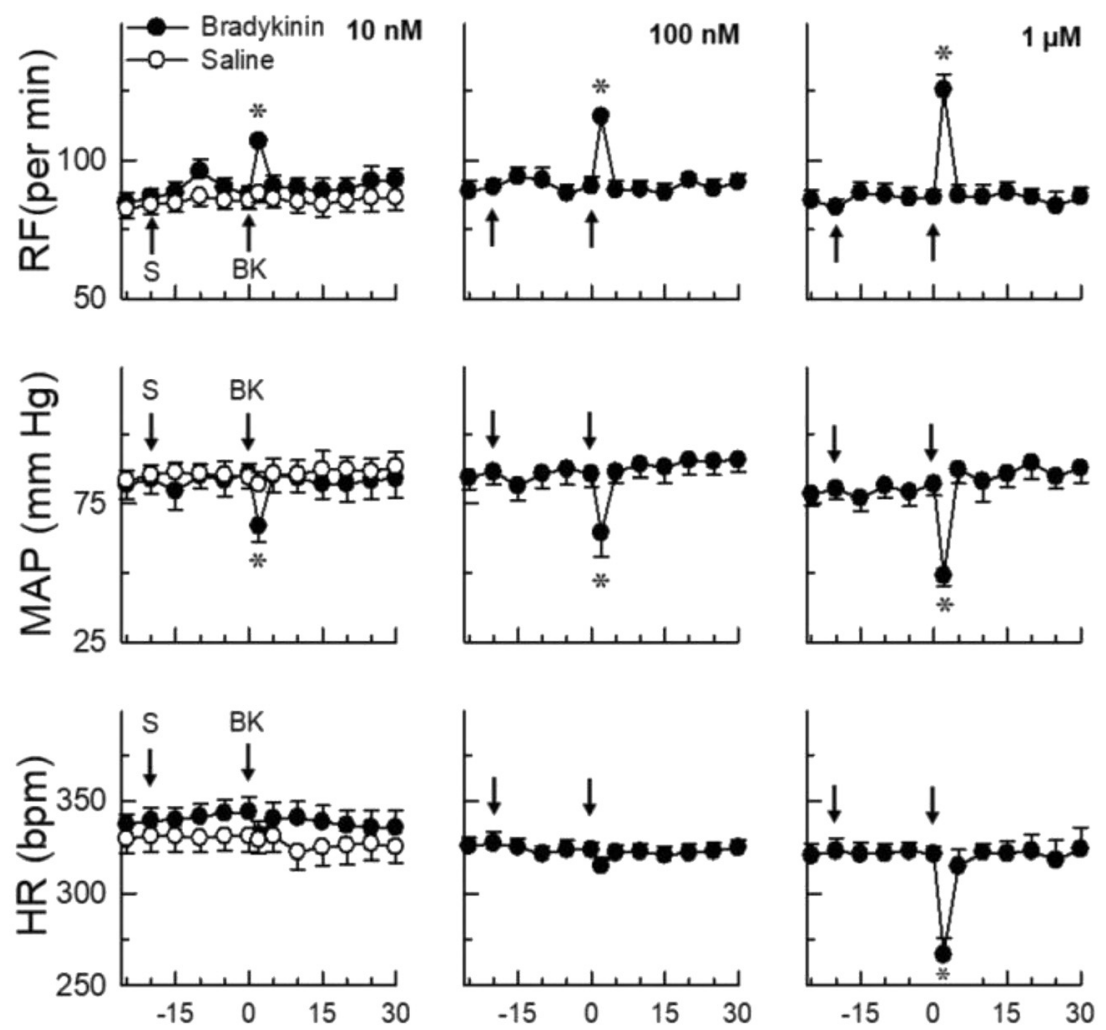

Time (min)

Figure 3. Time-response relationships of different concentrations of bradykinin on respiratory frequency (RF), mean arterial pressure (MAP) and heart rate (HR). The time-matched control responses after saline are given in the panels for $10 \mathrm{nM}$ concentration of bradykinin. The values are in mean \pm SEM from 6 experiments for each concentration. The responses after bradykinin are significantly different from the saline control at all concentrations $[P<0.05$, Post-Hoc correction using Dunnett's $t$-test (two sided)] except for the HR responses at $10 \mathrm{nM}$ and $100 \mathrm{nM}$ of bradykinin. An asterisk $\left(^{*}\right)$ indicates significant difference of responses compared to saline group. An arrow indicates the point of injection of saline (S)/bradykinin (BK)

\section{Effect of bradykinin on mean arterial pressure}

Bradykinin-induced changes in MAP were also concentration-dependent, minimal with $10 \mathrm{nM}$ and maximal with $1 \mu \mathrm{M}$ of concentration. After injection of bradykinin, there was an immediate fall in MAP (15-30 mm Hg) at all concentrations of bradykinin with a latency of 5-8 s; the value of MAP returned to the initial level within $5 \mathrm{~min}$ and remained at that level up to $30 \mathrm{~min}$. The 
decrease in MAP was significantly greater than in the time-matched saline group $[P<0.05$, PostHoc correction using Dunnett's $t$-test (two sided)] or as compared to the corresponding initial value at all concentrations of bradykinin $(P<0.05$, Student's $t$-test for paired observations). The optimal effect on MAP was observed with $1 \mu \mathrm{M}$ concentration of bradykinin (Figs 2 and 3 ).

\section{Effect of bradykinin on heart rate}

After i.a. injection of lower concentrations (10 and $100 \mathrm{nM}$ ) of bradykinin, there was a decrease in $\mathrm{HR}$ which was not different from the effect of saline. But at $1 \mu \mathrm{M}$ concentration of bradykinin, there was immediate bradycardiac response with a latency of $5 \mathrm{~s}$, which returned to the initial level within $5 \mathrm{~min}$ and remained at that level up to $30 \mathrm{~min}$. This decrease in HR (50-80 bpm) was significantly greater than in the time-matched saline group $[P<0.05$, Post-Hoc correction using Dunnett's $t$-test (two sided)] or as compared to the corresponding initial value at $1 \mu \mathrm{M}$ concentration of bradykinin $(P<0.05$, Student's $t$-test for paired observations). Since bradykinin-induced bradycardiac responses at lower concentrations were similar to the saline control responses, a higher concentration $(1 \mu \mathrm{M})$ of bradykinin was chosen as an optimal concentration for further experiments.

\section{Effect of bradykinin on latency of the cardiorespiratory parameters}

The time lag of the response (latency) was different for different cardiorespiratory parameters at different concentrations, but it was the shortest for $1 \mu \mathrm{M}$ concentration of bradykinin for all parameters. The latencies for tachypnea were $8.25 \pm 0.40 \mathrm{~s}, 5.50 \pm 0.50 \mathrm{~s}$ and $4.92 \pm 0.35 \mathrm{~s}$ for $10 \mathrm{nM}, 100 \mathrm{nM}$ and $1 \mu \mathrm{M}$ concentration of bradykinin, respectively. The latencies for hypotension were $8.00 \pm 0.34 \mathrm{~s}, 5.42 \pm 0.20 \mathrm{~s}$ and $5.00 \pm 0.13 \mathrm{~s}$ for $10 \mathrm{nM}, 100 \mathrm{nM}$ and $1 \mu \mathrm{M}$ concentration of bradykinin, respectively. The latencies for bradycardiac changes were $8.67 \pm$ $0.42 \mathrm{~s}, 6.83 \pm 0.40 \mathrm{~s}$ and $4.95 \pm 0.24 \mathrm{~s}$ for $10 \mathrm{nM}, 100 \mathrm{nM}$ and $1 \mu \mathrm{M}$ concentration of bradykinin, respectively.

\section{Effect of bradykinin on water content of hind paw}

The mean \pm SEM values of water content in the ipsilateral (bradykinin injected side) and contralateral (control) hind paws are given in Fig. 4 . The water content (\% of wet weight) on the ipsilateral side was not different from the contralateral side with saline, $10 \mathrm{nM}$ and $100 \mathrm{nM}$ concentration of bradykinin ( $P>0.05$, Student's test for paired observations). However, at $1 \mu \mathrm{M}$ concentration of bradykinin, the water content on the ipsilateral side was significantly greater than on the contralateral side $(P<0.05$, Student's $t$-test for paired observations). This indicates the presence of paw edema on the ipsilateral side at $1 \mu \mathrm{M}$ concentration of bradykinin.

The effect of bradykinin on all parameters was greater with $1 \mu \mathrm{M}$ concentration of bradykinin. Therefore, this concentration was chosen for subsequent sets of experiments for the elicitation of reflex responses.

\section{DICLOFENAC SODIUM PRETREATMENT BLOCKED BRADYKININ-INDUCED CHANGES}

Injection of diclofenac sodium ( $1 \mathrm{mg} / \mathrm{kg}$, i.a.) per se did not produce any change in the resting RF, MAP and $\mathrm{HR}$ up to $20 \mathrm{~min}$. However, diclofenac sodium pretreatment 


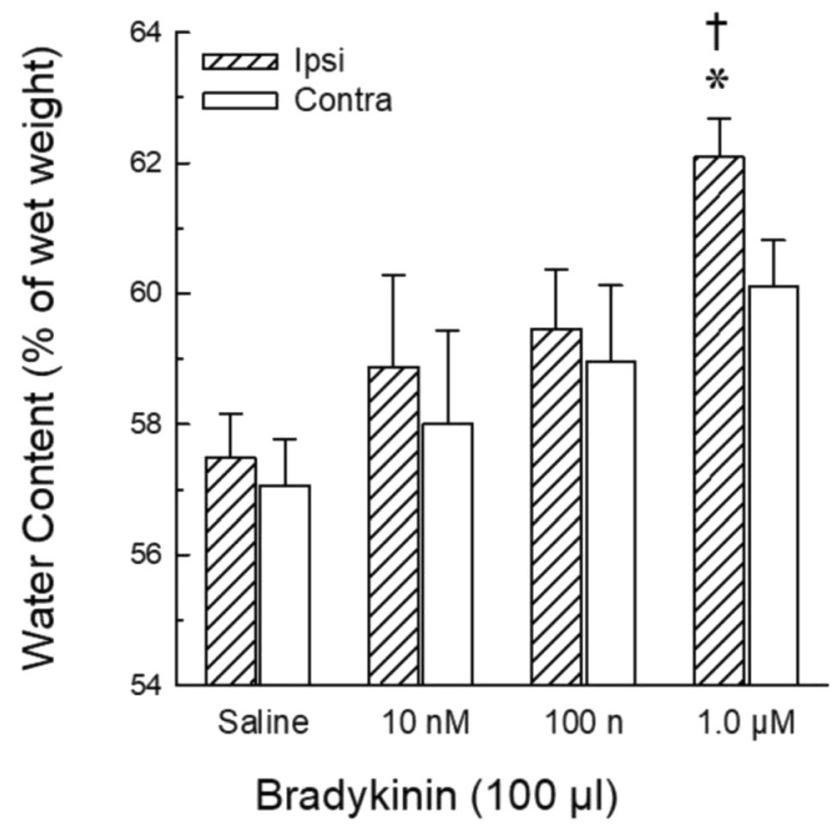

Figure 4. Histograms showing the water content of ipsilateral (Ipsi) and contralateral (Contra) hind paws after i.a. injection of different concentrations of bradykinin, with the volume of injectables kept constant $(0.10 \mathrm{~mL})$. The values are mean \pm SEM from 6 experiments for each concentration. An asterisk $(*)$ indicates significant difference from contralateral side $(P<0.05$, Student's $t$-test for paired observations) and $(\dagger)$ indicates significant difference from the corresponding paw in saline only group $[P<0.05$, Post-Hoc correction using Dunnett's $t$-test (two sided)]

markedly attenuated the tachypneic changes (RF from $45 \%$ to $7 \%$ ), hypotensive changes (MAP from $40 \%$ to $11 \%$ ) and bradycardiac changes (HR from $17 \%$ to $2 \%$ ) produced by bradykinin, at a significant level [Fig. 5; $P<0.05$, Post-Hoc correction using Dunnett's $t$-test (two sided)].

The data of water content in the ipsilateral and contralateral hind paws is given in the histograms (Fig. 5D). In diclofenac-pretreated animals, edema was not observed in the ipsilateral hind paw as compared to the contralateral hind paw $(P>0.05$, Student's $t$-test for paired observations).

\section{NEUROTOMY ATTENUATED BRADYKININ-INDUCED CHANGES}

Neurotomy (ipsilateral femoral and sciatic nerve sectioning) per se did not produce any change in the resting RF, MAP and HR up to 15 min. However, neurotomy attenuated the tachypneic changes (RF from $45 \%$ to $13 \%$ ), hypotensive changes (MAP from $40 \%$ to $18 \%$ ) and bradycardiac changes (HR from $17 \%$ to $4 \%$ ) produced by bradykinin, at a significant level (Fig. $6 ; P<0.05$, 

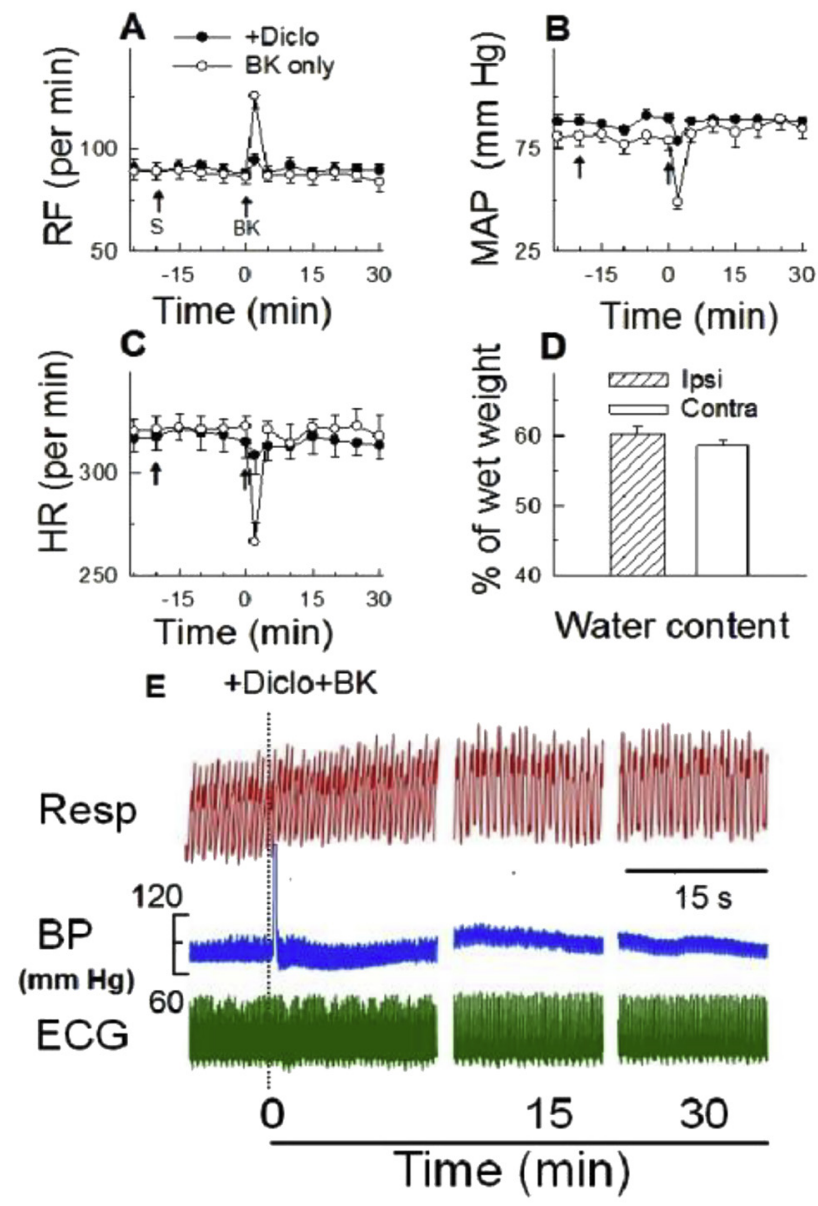

Figure 5. Diclofenac sodium (+Diclo) pretreatment blocked the bradykinin-induced responses. The time-matched response relationship in diclofenac sodium pretreated animals $(n=6)$ and bradykinin $(\mathrm{BK})$ only group in respiratory frequency (RF), mean arterial pressure (MAP) and heart rate (HR) [Fig. A-C] are shown. The RF, MAP and HR responses are significantly different from the bradykinin only group $[P<0.05$, Post-Hoc correction using Dunnett's $t$-test (two sided)]. An arrow indicates the point of injection of saline (S)/bradykinin (BK). Histogram (D) shows the water contents of ipsilateral and contralateral hind paws in diclofenac-pretreated animals and were found not to be different from each another $(P>0.5$, Student's $t$-test for paired observations). Figure "E" displays the actual recordings showing the effect of bradykinin ( $1 \mu \mathrm{M}$, i.a.)-induced changes on respiration (Resp), blood pressure (BP) and electrocardiogram (ECG) in diclofenac-pretreated animals as compared to the bradykinin only group. Dotted line indicates the point of injection of bradykinin in original tracing. The horizontal line indicates $15 \mathrm{~s}$ for all parameters

Post-Hoc correction using Dunnett's $t$-test [two sided]). The RF, MAP and HR changes were similar in direction but markedly attenuated, and remained more or less at the initial level up to $30 \min$ (Fig. 6). 

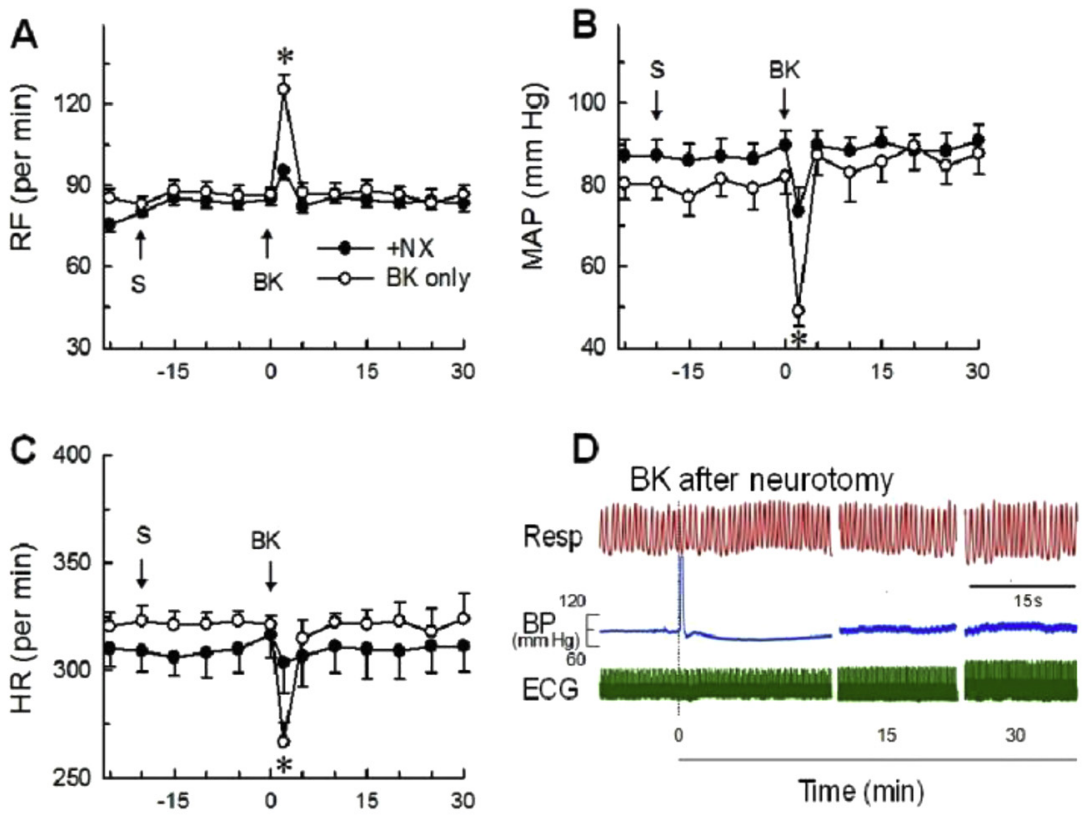

Figure 6. Neurotomy $(+\mathrm{NX})$ pretreatment blocked the bradykinin-induced responses. The time-matched response relationship in neurotomized animals $(n=6)$ and bradykinin (BK) only group in respiratory frequency (RF), mean arterial pressure (MAP) and heart rate (HR) [Fig. A-C] are shown. The RF, MAP and $\mathrm{HR}$ responses are significantly different from the bradykinin only group $[P<0.05$, Post-Hoc correction using Dunnett's $t$-test (two sided)]. An arrow indicates the point of injection of saline (S)/bradykinin (BK). Figure " $D$ " indicates the actual recordings showing the effect of bradykinin $(1 \mu \mathrm{M}$, i.a.)-induced changes on respiration (Resp), blood pressure (BP) and electrocardiogram (ECG) in neurotomized animals as compared to the bradykinin only group. Dotted line indicates the point of injection of bradykinin in the original tracing. The horizontal line indicates $15 \mathrm{~s}$ for all parameters

\section{DISCUSSION}

The present observations reveal that instillation of bradykinin into a segment of the femoral artery evoked reflex cardiorespiratory changes, whereas in control experiments equi-volume of saline did not, excluding the possibility of stretch/ischemia-induced responses of the vessel wall. The cardiorespiratory changes were observed almost simultaneously as tachypneic, hypotensive and bradycardiac responses with a latency of 5-8 s. In addition, paw edema was also observed in the hind limb on the ipsilateral side. All the responses were significantly attenuated after diclofenac pretreatment or ipsilateral neurotomy.

It is reported elsewhere that injection of nociceptive agents (capsaicin, anandamide and $\alpha, \beta$-MeATP) into the right common iliac artery, approached from the left femoral artery via a catheter produced cardiorespiratory changes mediating perivascular afferents [18, 27]. In another study from our laboratory, it has been shown that i.a. injection of BT venom elicits cardiorespiratory reflexes involving perivascular afferents [25]. In the present study, the 
methodology was further modified by injecting drugs (saline/agonist/antagonists) into the right-side femoral artery by using a pediatric i.v. cannula with injection valve $(24 \mathrm{G}$, double ported). This experimental design provided us an opportunity to demonstrate the effect of nociceptive agents on cardiorespiratory parameters prevailing in a local segment of an artery. The time-matched saline control (Figs 2 and 3) was used to eliminate the possibility of local ischemic/stretch-induced responses modulating the cardiorespiratory reflexes. Bradykinin was used as a pure nociceptive agent for the elicitation of reflex responses. The bradykinininduced cardiorespiratory changes are unlikely to be due to systemic spillage, as bradykinin was instilled retrogradely in an artery, and the latency of responses is also shorter (5-8 s) favoring the neural mechanism of responses. Since the cannulated artery was turned into an end artery, therefore the possibility of spillage of chemicals into the systemic circulation becomes negligible. Furthermore, the volume of drugs was kept at minimum $(0.10 \mathrm{~mL})$ and constant for all the chemicals to avoid the systemic spillage and stretch-induced responses, if any.

The responses observed on RF and MAP were concentration-dependent, as the tachypneic and hypotensive responses were minimal at $10 \mathrm{nM}$ and maximal at $1 \mu \mathrm{M}$ concentration of bradykinin. However, bradycardiac responses at lower concentrations (10 $\mathrm{nM}$ and $100 \mathrm{nM}$ ) were not different from the saline control responses and maximal at higher concentration (1 $\mu \mathrm{M})$, indicating the relationship between the strength of stimulus and the responses it produced. The effect of $1 \mu \mathrm{M}$ concentration of bradykinin on all parameters was greater, therefore this concentration was chosen for subsequent sets of experiments for elicitation of reflex responses.

Bradykinin is produced in a variety of conditions like inflammation, ischemia, infarction, hypoxia, lactic acidosis etc., and can stimulate the sensory nerve endings modulating the cardiorespiratory responses [17]. In a study elsewhere, it has been shown that bradykinin and capsaicin produces reflex cardiorespiratory responses through stimulation of hind limb muscle $[15,22]$. In the present study, it is shown that i.a. injection of bradykinin produces immediate tachypneic and hypotensive responses at lower concentrations (10 nM and $100 \mathrm{nM})$ and immediate tachypneic, hypotensive and bradycardiac responses at higher concentrations (1 $\mu \mathrm{M})$ of bradykinin with shorter latencies of responses (5-8 s). In this study, the pattern of changes in BP, HR and RF are physiological, as there are hypotensive, bradycardiac and hyperventilatory responses, respectively, after injection of a nociceptive agent $[25,28]$. However, we are not able to identify the exact anatomical location of sensory nerve endings in this study; therefore, the possible involvement of afferents located elsewhere cannot be ignored.

Paw edema observed at $1 \mu \mathrm{M}$ concentration on the ipsilateral side can be attributed to the direct effect of bradykinin on the capillary endothelium or to activation of the release of inflammatory mediators in the surrounding tissues. In earlier works from this laboratory, the action of venom is shown to involve kinins $[2,3,7,8]$. Kinins are known to mediate their actions via prostaglandins [6]. It was also reported that indomethacin pretreatment blocked the venominduced vasosensory reflex responses [25]. In this study, diclofenac sodium pretreatment blocked the bradykinin-induced paw edema and also blocked the reflex cardiorespiratory changes. Thus, observations suggest that prostaglandins (PGs) are involved in producing the vasosensory reflex mediated changes in the RF, MAP and HR responses. It is well-known that PGs augment the release of chemicals/transmitters from the nerve terminals and also increase 
capillary permeability to produce edema [14]. Furthermore, PGs sensitize the peripheral nociceptors by lowering the firing threshold of nociceptive sensory neurons [11, 12, 20, 23]. Thus, it seems that there is a link between nociception and paw edema.

It has been shown by some researchers that ipsilateral femoral and sciatic nerve sectioning results in the attenuation of vasosensory reflex responses [25, 27]. Our findings are consistent with the above observations and demonstrate that ipsilateral femoral and sciatic nerve sectioning attenuates all the bradykinin-induced cardiorespiratory responses (MAP, RF and HR) significantly, indicating that most parts of the afferents are located within these somatic nerves. Attenuation of responses after denervation also supports our assumption that the responses are mediated by perivascular nerves and are not due to other reasons.

\section{CONCLUSION}

In conclusion, the presence of bradykinin in a segment of peripheral artery evokes cardiorespiratory changes as equi-volume of saline does not, excluding the possibility of ischemia/stretch-induced responses of the vessel wall. The cardiorespiratory changes were observed almost simultaneously as tachypneic, hypotensive and bradycardiac responses of a shorter latency (5-8 s), indicating the neural mechanisms of responses. In addition, paw edema was observed on the ipsilateral side. Pretreatment with diclofenac sodium blocked all the cardiorespiratory responses as well as the ipsilateral paw edema, indicating the role of PGs in producing the responses. Attenuation of responses after neurotomy also supports our proposition that the responses originate from the local vascular bed involving perivascular afferents, and are not due to systemic spillage of bradykinin. Our data support the hypothesis regarding the role of peripheral blood vessels in the regulation of cardiorespiratory responses.

\section{ACKNOWLEDGMENTS}

Declared none.

\section{REFERENCES}

[1] Achyuthan KE, Agrawal OP, Ramachandran LK. Enzymes in the venoms of two species of Indian scorpion. Indian J Biochem Biophys. 1982; 19: 356-8.

[2] Bagchi S, Deshpande SB. Indian red scorpion (Buthus tamulus) venom-induced augmentation of cardiac reflexes is mediated through involvement of peripheral $5-\mathrm{HT}_{3}$ and central $5-\mathrm{HT}_{1 \mathrm{~A}}$ receptor subtypes. Toxicon 1999; 37: 1697-709.

[3] Bagchi S, Deshpande SB. Indian red scorpion venom-induced augmentation of cardiac reflexes is mediated through the mechanisms involving kinins in urethane anesthetized rats. Toxicon 1998; 36: 309-20.

[4] Basu A, Gomes A, Dasgupta SC, Lahiri SC. Histamine, 5-HT and hyaluronidase in the venom of the scorpion Lychas laevifroms (Pock). Ind J Med Res. 1990; 92: 371-3. 
[5] Chhatwal GS, Habermann E. Neurotoxin, protease inhibitors and histamine releaser in the venom of Indian red scorpion (Buthus tamulus): Isolation and partial characterization. Toxicon 1981; 19: 807-23.

[6] Davis AJ, Perkin MN. The involvement of bradykinin $B_{1}$ and $B_{2}$ receptor mechanisms in cytokine-induced mechanical hyperalgesia in rat. Br J Pharmacol. 1994; 113: 63-8.

[7] Deshpande SB, Alex AB, Jagannadham MV, Rao GRK, Tiwari AK. Identification of a novel pulmonary edema producing toxin from Indian red scorpion (Mesobuthus tamulus) venom. Toxicon 2005; 45: 735-43.

[8] Deshpande SB, Bagchi S, Rai OP, Aryya NC. Pulmonary oedema produced by scorpion venom augments phenylediguanide-induced reflex response in anesthetized rats. J Physiol. 1999; 521: 537-44.

[9] Donnerer J, Lembeck F. Analysis of the effects of intravenously injected capsaicin in the rat. Naunyn Schmiedebergs Arch Pharmacol. 1982; 320: 54-7.

[10] Donnerer J, Lembeck F. Capsaicin-induced reflex fall in rat blood pressure is mediated by afferent substance P-containing neurons via a reflex centre in the brainstem. Naunyn Schmiedebergs Arch Pharmacol. 1983; 324: $293-5$.

[11] Evans AR, Junger H, Michael DS, Nicol GD, Linda SS, Broome JT, et al. Isoprostanes, novel eicosanoids that produce nociception and sensitize rat sensory neurons. J Pharmacol Exp Ther. 2000; 293: 912-20.

[12] Ferreira SH, Nakamura M, de Abreu Castro MS. The hyperalgesic effects of prostacyclin and prostaglandinE2. Prostaglandins 1978; 16: 31-7.

[13] Galvez A, Gimnez-Gallego G, Reuben JP, Roy Contancin L, Fiegenbaun P, Kaczorowsky J, et al. Purification and characterisation of a unique, potent peptidyl probe for the high conductance calcium-activated potassium channel from venom of the scorpion Buthus tamulus. J Biol Chem. 1990; 285: 11083-90.

[14] Julieus D, Basabaum AI. Molecular mechanism of nociception. Nature. 2001; 413: 203-10.

[15] Kaufman MP, Iwamoto GA, Longhurst JC, Mitchell JH. Effects of capsaicin and bradykinin on afferent fibers with ending in skeletal muscle. Circ Res. 1982; 50: 133-9.

[16] Kilo S, Berghoff M, Hilz M, Freeman R. Neural and endothelial control of the microcirculation in diabetic peripheral neuropathy. Neurology 2000; 54: 1246-52.

[17] Marshall JN. Peripheral Chemoreceptors and Cardiovascular regulations. Physiol Rev. 1994; 74: 543-94.

[18] McQueen DS, Bond SM, Moores C, Chessell L, Humphrey PPA, Dowd E. Activation of P2X receptors for adenosine triphosphate evokes cardiorespiratory reflexes in anesthetized rats. J Physiol. 1998; 507: 843-55.

[19] Nassar AY, Abu-Sinna G, Soliman FM, Rahim SA, El-Saddan IM. Bradykinin potentiating fraction, isolated from venom of Buthus occitanus promotes spermatogenesis in premature mice. In: Gopalakrishnakone P, Tan CK, editors. Recent advances in toxicology research. Singapore: Venom and Toxin Research Group, National University; 1992, vol. 2, pp. 118-38.

[20] Nicol GD, Cui M. Prostaglandin-E2 enhances bradykinin inactivation of embryonic rat sensory neurons. J Physiol (Lond). 1994; 480: 485-92.

[21] Pedarzani PD, Hoedt D, Doorty KB, Wadswarth JDF, Joseph JS, Jeyaseelan K, et al. Strong PN: Tamapin, a venom peptide from the Indian red scorpion (Mesobuthus tamulus) that targets small conductance $\mathrm{Ca}^{+}$ activated $\mathrm{K}^{+}$channels and after hyperpolarization currents in central neurons. J Biol Chem. 2002; 277: 46101-9.

[22] Sato A, Sato Y, Schmidt RF. Changes in heart rate and blood pressure upon injection of algesic agents in to skeletal muscle. Pflugers Arch. 1982; 393: 31-6.

[23] Schaible HG, Schmidt RF. Excitation and sensitization of fine articular afferents from cats knee joint by prostaglandin-E2. J Physiol (Lond). 1988; 413: 91-104.

[24] Singh SK, Deshpande SB. Injection of Mesobuthus tamulus venom in distal segment of femoral artery evokes hyperventilatory and hypertensive responses in anesthetised rats. Neurosci Lett. 2008a; 438: 64-6. 
[25] Singh SK, Deshpande SB. Intra-arterial injection of Mesobuthus tamulus venom elicits cardiorespiratory reflexes involving perivascular afferents. Toxicon 2005; 46: 820-6.

[26] Singh SK, Deshpande SB. Vasosensory responses elicited by Indian red scorpion venom last longer than capsaicin-induced responses. Ind J Exp Biol. 2008b; 46: 755-9.

[27] Smith PJW, McQueen DS. Anandamide induces cardiovascular and respiratory reflexes via vasosensory nerves in anesthetized rat. Br J Pharmacol. 2001; 134: 655-63.

[28] Smith PJW, McQueen DS. Perivascular nerves induce cardiorespiratory reflexes in response to algogens in anesthetized rats. Neurosci Res. 2004; 50: 271-81.

[29] Stansberry KB, Peppard HR, Babyak LM, Popp G, McNitt PM, Vinik AI. Primary nociceptive afferents mediate the blood flow dysfunction in non-glabrous (hairy) skin of type 2 diabetes: a new model for the pathogenesis of microvascular dysfunction. Diabetes Care 1999; 22: 1549-54.

[30] Wudayagiri R, Inceoglu B, Herrmann R, Derbel M, Choudary PV, Hemmock BD. Isolation and Characterization of a novel lepidopteran- selective toxin from the venom of South Indian red scorpion, Mesobuthus tamulus, BMC Biochemistry 2001; 2: 16-28. 